\title{
Social vs. Nonsocial Housing Differentially Affects Perseverative Behavior in Rats (Ratus norvegicus)
}

\author{
Brittany M. Hemmer ${ }^{1}$, Audrey E. Parrish ${ }^{2}$, Taylor B. Wise ${ }^{1}$, Marc Davis ${ }^{1}$, Margaret Branham ${ }^{1}$, \\ Dominique E. Martin ${ }^{1}$, and Victoria L. Templer ${ }^{*}$
}

${ }^{1}$ Department of Psychology, Providence College, Providence, RI USA

${ }^{2}$ Department of Psychology, The Citadel, Charleston, SC USA

*Corresponding author (Email: vtempler@providence.edu)

Citation - Hemmer, B. M., Parrish, A. E., Wise, T. B., Davis, M., Branham M., Martin, D. E., \& Templer, V. L. (2019). Social vs. nonsocial housing differentially affects perseverative behavior in rats (Ratus norvegicus). Animal Behavior and Cognition, 6(3), 168-178. https://doi.org/10.26451/abc.06.03.02.2019

\begin{abstract}
Perseverance, also commonly referred to as grit or industriousness, is the continued effort exerted to complete goal-directed tasks. Many factors, such as stress, can contribute to perseverative behavior, but the role of sociality on perseverance in animal models has not been studied. In this experiment, perseverance was measured in Long-Evans rats; half of which were socially housed ( $\mathrm{SH}$ ) and the other half were nonsocially housed (NSH). Rats were placed in a continuous T-maze; one arm of the maze contained an unobstructed low value reward and the other arm contained a high value reward blocked by a barrier that progressively increased in height across testing sessions. We will hereon refer to the low value reward and high value reward as the low reward and the high reward, respectively. Perseverative behavior was assessed by time spent interacting with the barrier and trials were characterized as either adaptive perseverative trials (high reward obtainment) and maladaptive perseverative trials (low reward obtainment after abandoning attempts to overcome the high reward barrier). SH and NSH rats were equally proficient at overcoming a physical barrier to obtain a higher-valued reward, but the NSH rats spent more time interacting with the barriers during maladaptive perseverative trials than SH rats. NSH rats thus exhibited prolonged efforts to overcome the barrier only to ultimately travel to the low reward option. In contrast, SH rats selected the low reward option earlier in the trial and did not maladaptively perseverate without obtaining the high reward. Putative evidence for increased perseverance in NSH rats is explained in the context of maladaptive perseverative behavior rather than perseverance per se. Increased adaptability and acquisition of task-set in SH rats suggests a role of social housing in advantageous decision making.
\end{abstract}

Keywords - Perseverance, Enrichment, Rats, Industriousness, Grit, Acquisition. Social Housing

The term perseverance has been referred to as industriousness, grit, persistence, or achievement orientation in the human literature. Though subtle differences in these personality characteristics exist, these traits have all been described as the continuation of exerted effort to complete goal-directed tasks (e.g., Duckworth, Peterson, Matthews, \& Kelly, 2007; Eisenberger, 1992; Feather, 1962; Williams \& DeSteno, 2008). Hereon, we will refer to this behavior of interest as perseverance. Perseverance has become a topic of research in the human literature (e.g., Charney, 2004; Feder, Nestler, \& Charney, 2009; Russo, Murrough, Han, Charney, \& Nestler, 2012) with much of it addressing how stress, trauma, early life experience, and other factors lead to changes in behavior (e.g., Heim, Newport, Mletzko, Miller, \& Nemeroff, 2008; Rasmusson, Vythilingam, \& Morgan, 2003). Due to the various positive life outcomes associated with high levels of perseverance in humans, such as life satisfaction and success, this topic has become very popular in recent decades (Duckworth et al., 2007; Eisenberger, 1992; Feather, 1962; Feder 
et al., 2009; Russo et al., 2012). However, outcomes in human studies often are confounded by myriad variables, including various life experiences, stress, and other personality factors that may influence perseverance (Credé et al., 2017). For these reasons, animal models of perseverance could prove particularly useful as they afford a high degree of experimental control such that factors affecting perseverative behavior can be better isolated.

Researchers have recently begun to fill this gap using animal models with efforts to isolate the mechanisms behind perseverative behavior divorced of confounding factors (e.g., Lambert et al., 2006; Laurence, Labuschagne, Lura, \& Hillman, 2015). For example, Laurence et al. (2015) demonstrated that regular exercise enhances task-based "industriousness" in laboratory rats. A continuous T-maze in which one arm, with no barrier, contained a low reward and the other arm contained a high reward blocked by a climbing barrier, was utilized. Rats on an exercise regimen exhibited perseverative behavior by selecting a high-effort/high reward option more often than control rats. Here, perseverative behavior is a behavioral outcome in which effort is exerted to complete a goal-directed task. In line with this operational definition, we will hereon refer to perseverative behavior when we refer to the observable effort expended to complete a task. In another study, Lambert et al. (2006) investigated coping strategies in response to stress in Long-Evans rats. Experimental worker rats were trained to dig for food rewards in an open field covered with corncob bedding, whereas control rats received the same rewards regardless of their physical effort. Rats were then exposed to a novel plastic cat toy containing a bell and food reward. The worker rats persisted longer in efforts to remove the reward as compared with the control rats. The results of these experiments indicate that acquired behavioral life experiences contribute to perseverance during times of challenge.

Although perseverance is often described as a positive trait due to its associations with success, positive emotionality, and industriousness (Garcia, 2011; Laurence et al., 2015), perseverance also may be considered negative or maladaptive. Specifically, perseverative behavior also may result in negative and costly behaviors when an individual persists too long on challenging tasks rather than favoring simpler, more solvable problems (e.g., Cloninger, Zohar, Hirschmann, \& Dahan, 2011; Lucas, Gratch, Cheng, \& Marsella, 2015). One classic example of this is in the form of perseverative errors, which are commonly assessed in the human and comparative psychology literature. The midsession reversal (MSR) task, in which reward contingencies for a two-choice discrimination (S+/S-) are reversed (S-/S+) at a particular point during the test session, is often used to assess erroneous perseverance (see RayburnReeves \& Cook, 2016 for a review). Perseverative errors provide a useful way of observing perseverative behavior through incorrect choices for previously reinforced stimuli despite a current lack of reinforcement. Perseverative behavior can also be observed by examining costly persistence as opposed to erroneous persistence. For example, Lucas, et al. (2015) found that human participants who scored higher on a grit scale were more likely to increase effort when they were failing and persist even if they would incur a cost for their perseverance. Perseverance in this task was not incorrect because reinforcement was present if efforts were successful; however, subjects' persistence was nevertheless maladaptive because of awareness that success was unlikely. Thus, some studies have begun to dissociate the positive and negative aspects of perseverance; however, more research is necessary to reconcile these conflicting outcomes of perseverative behaviors.

One of the factors that could influence perseverance in laboratory animals is environmental enrichment (EE). EE and social housing have been shown to positively benefit cognition in laboratory rats (Simpson \& Kelly, 2011). Social housing is often a key component of laboratory rat enrichment, because acute isolation in rats can lead to severe cognitive deficits (Fone \& Porkness, 2008). However, laboratory animals often are not socially housed or given enrichment during experiments. The individual effects of social housing, divorced from EE such as exercise, have not been studied in depth. Recent work from our laboratory demonstrated that in rats, social housing, independent of other forms of enrichment, is associated with positive cognitive benefits during different life stages (Heimer-McGinn, Wise, Hemmer, Dayaw, \& Templer, submitted; Templer, Wise, \& Heimer-McGinn, 2019). When tested on different versions of the Barnes Maze (BM), socially-housed ( $\mathrm{SH}$ ) rats exhibited enhanced acquisition of the taskset and quicker reversal learning as compared with nonsocially-housed rats (NSH; Heimer-McGinn et al., 
submitted). When fully aged, SH rats also demonstrated fewer working memory errors in the radial armmaze as compared with their NSH counterparts (Templer et al., 2019). This demonstrates that social housing promotes cognitive adaptability, spatial learning in adulthood, and protects against age-related deficits in working memory. Due to our recent work suggesting that social housing is associated with cognitive benefits (Heimer-McGinn et al., submitted; Templer et al., 2019), it is worth examining whether the benefits of social housing also extend to influence perseverance. Further, due to our findings that social housing offers a protective benefit against age-related cognitive decline, it is important to investigate how social enrichment, aging, and perseverance simultaneously interact. This information may prove particularly useful to those studying aging human populations, as social behavior in old age is beneficial for overall health (Domènech-Abella et al., 2017; Mukerjee, 2013). Specifically, we studied the impact of social housing on perseverance with specific attention to adaptive perseverance (i.e., obtaining a successful outcome by expending effort) - versus over-perseverance (maladaptive behavior, i.e., expending maximum effort on a task but ultimately abandoning attempts at success.

To test the role of social housing on perseverance, we modified the Laurence et al. (2015) paradigm, placing both SH and NSH rats in a continuous T-Maze. The maze consists of a central arm rats must traverse and ends in a right or left turn. The rats must choose to turn right or left and enter the chosen side arm to receive the respective reward. After retrieving the reward, the rats continue down the chosen side arm to re-enter the central arm and begin another trial (Figure 1). In the current experiment, one arm contained a low reward and no barrier, and the other arm contained a high reward blocked by a barrier that progressively increased in height across testing sessions. As the barrier height increased, obtaining the high reward became more difficult and therefore required more perseverative behavior to overcome.

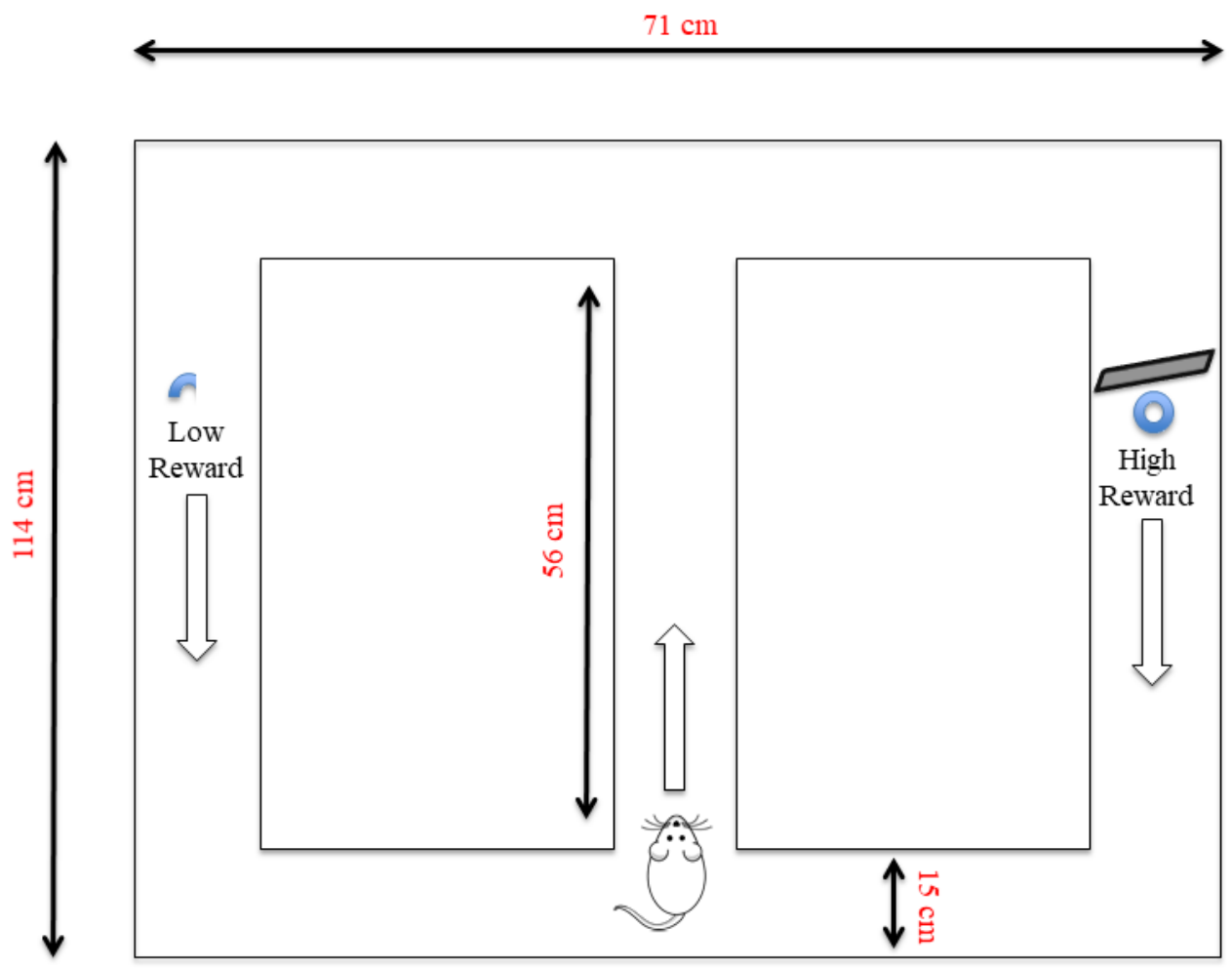

Figure 1. The continuous T-maze apparatus, with the rat depicted at the starting point, the high-reward was positioned behind a barrier in the left arm and the low-reward was positioned in the right arm (no barrier) for all trials. 
We examined the role of social housing on perseverative behavior by examining two behavioral measures. First, we analyzed success rates for each barrier height, which was defined as obtaining the high reward. Second, we operationally defined perseveration as time spent interacting with the barrier, so we examined the amount of time the rat came into contact with the barrier, including sniffing. We categorized perseverative behavior as either adaptive or maladaptive. Adaptive perseverance trials would result in increased time spent interacting with barriers that ultimately led to success in obtaining the high reward. Maladaptive perseverance would result in an increase in time spent interacting with barriers that ultimately led to failure in obtaining the high reward (i.e., too much time spent on a failed task; Figure 2). Because exerting efforts to overcome the barrier are indicative of perseverative behavior, trials in which the rat initially chose the low reward option would not be considered maladaptive or adaptive perseverance due to the lack of effort required in these trials (Figure 2).

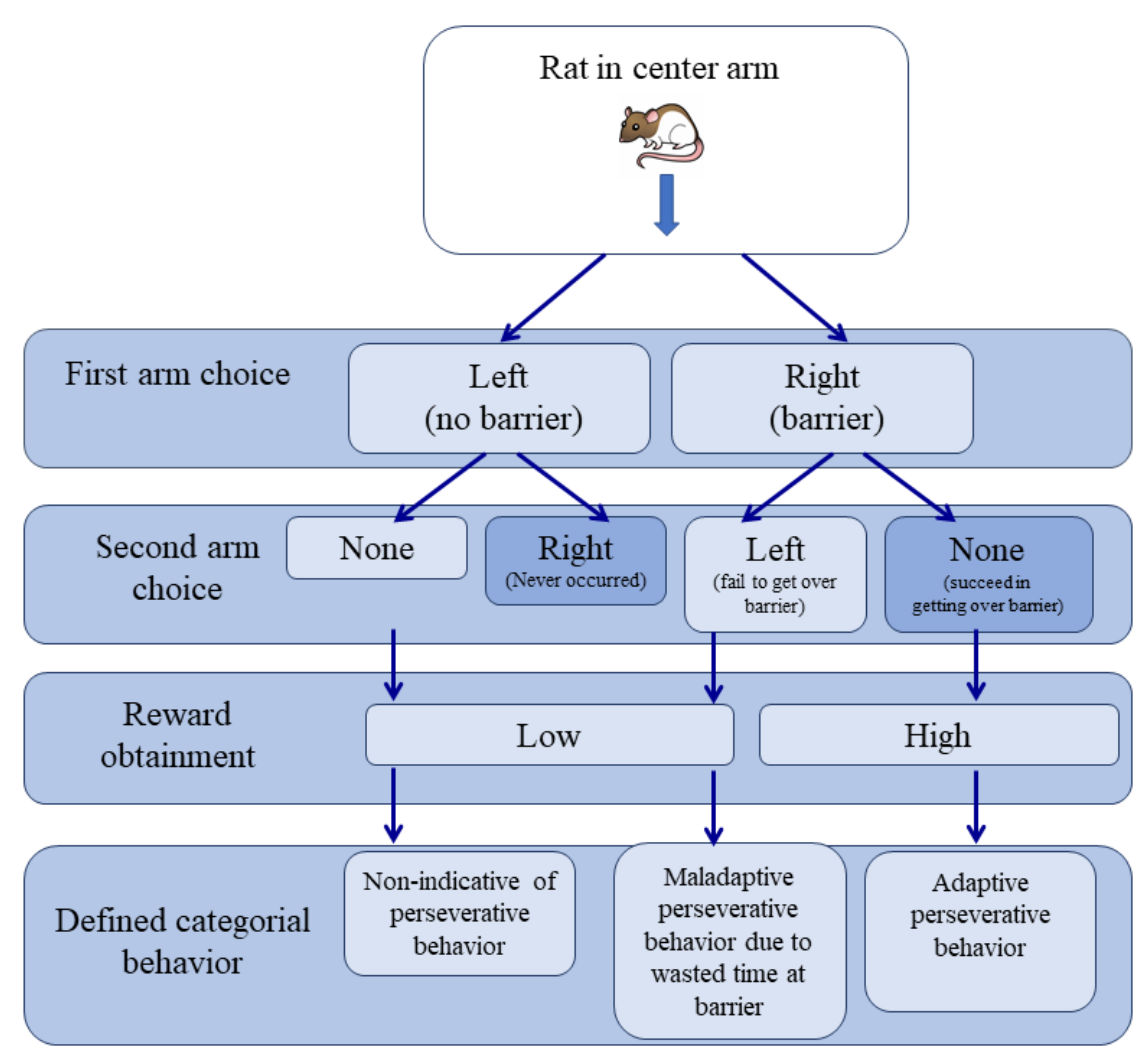

Figure 2. Flow chart illustrating defined categorical behavior based on a rat's arm choice (no barrier or barrier), if the rat switched arms, and the resulting reward obtainment.

\section{Method}

\section{Subjects}

We tested 19 male Long-Evans rats (Charles River Laboratories, Boston, MA, USA). Rats, born on the same day from separate litters, arrived on post-natal day (PND) 21, and were housed in the same colony room, maintained at $21.6^{\circ} \mathrm{C}$, and kept on a 12:12 reversed light: dark cycle, with light offset at $8 \mathrm{am}$ and light onset at $8 \mathrm{pm}$. Rats had ad libitum access to water but were food-restricted and maintained at $85-90 \%$ body weight after reaching adulthood (PND 110). Initially, the SH group was comprised of 10 rats, but one died of natural causes (PND 264). SH rats lived in a large, wire-mesh metal group cage $(91 \times 61 \times 160 \mathrm{~cm})$ and $10 \mathrm{NSH}$ rats also lived in large, wire-mesh metal cages but were individually housed $(81 \times 56 \times 53 \mathrm{~cm})$. All cages contained multiple platforms, corncob bedding, and a tray at the bottom (Ferret Nation). NSH rats had no physical contact with other rats but had visual and olfactory 
access to all other subjects. Both SH and NSH cages contained object enrichment, including platforms, running wheels, several plastic toys and enclosures (e.g., "igloos"), wooden chew toys, an open-topped plastic shoebox cage $(43 \times 20 \times 20 \mathrm{~cm})$. Subjects remained in their respective housing conditions for approximately two years, and testing began when rats were in late adulthood (PND 654). Subjects were tested on unrelated spatial and memory tasks throughout development, including the Barnes Maze, Radial Arm Maze, Open Field Test, and elevated Zero Maze; subjects had not previously been tested on the continuous T-maze as tested here. Both SH and NSH rats received the same task exposure (HeimerMcGinn et al., submitted; Templer et al., 2019; Templer, Wise, Dayaw, \& Dayaw, 2018). The experiments were carried out in accordance with NIH guidelines for the care and use of rats in research and all described in the current study were approved by the Institutional Animal Care and Use Committee (IACUC) of Providence College.

\section{Apparatus}

Multicolor plastic Jumbo LEGO blocks were used to construct a continuous T-maze with arms measuring $114 \times 71 \times 33 \mathrm{~cm}$ and $15 \mathrm{~cm}$ wide alleys for the rats to move in (Figure 1). Visual cues were displayed on both the left and the right wall of the T-maze to help spatially distinguish the two peripheral arms; a large circle indicated low reward and a series of diagonal lines indicated high reward. EthoVision XT software (Noldus Technologies) was used to record the trials to allow experimenters to clarify any behaviors that were unclear during the observation. For positive food reinforcement, a single piece of cereal (Froot Loop ${ }^{\circledR}$ ) constituted the high reward and a quarter piece of cereal was considered low reward. The respective reward was placed in each arm of the maze prior to the start of the trail and was replenished before the rat began the next trial. Rectangular barriers were constructed for three difficulty conditions: High Barrier: 10 × 5 x $23 \mathrm{~cm}$, Medium Barrier: 10 × 5 x $15 \mathrm{~cm}$, and Low Barrier: 10 × 5 × 8 $\mathrm{cm}$. The apparatus, including the barriers, was cleaned with $70 \%$ isopropyl alcohol between each rat to eliminate olfactory cues.

\section{Design and Procedure}

Training. Rats underwent two consecutive sessions of training on separate days (10 trials/session) to ensure that they were exposed to both sides of the maze containing the respective reward (right arm - high reward; left arm - low reward). At trial onset, a rat was placed in the central arm of the T-maze with no barriers present in either peripheral arm. Training served the dual purpose of ensuring that rats preferred the high reward to the low reward because both rewards were unobstructed by a barrier. The rat was given an unlimited amount of time to choose an arm of the maze. After an arm was chosen, if the rat began to turn in the opposite direction, a plastic partition was gently placed behind the rat to block it from running down the unchosen arm or back down the center arm. To begin the next trial, the rat was required to enter the center arm of the T-maze by first traversing one of the far lateral walls. As the rat entered the center arm of the T-maze, researchers replenished the consumed reward from the previous trial and the next trial began immediately.

If the rat did not choose either arm of the continuous T-maze for at least five trials over the course of training, four forced trials were conducted. By using a plastic partition to block entry to the low reward arm, two of these forced trials required the rats to select the high reward arm. The other two forced trials required the rats to go to the low reward arm by using a plastic partition to block the high reward arm. This ensured that rats experienced exposure to both the high and low reward at least twice.

Testing. Rats completed three consecutive test sessions on separate days (10 trials/session) for a total of 30 trials per rat. Thus, all testing was complete after three testing days. A different barrier height was presented on the high reward side for each test session (Session 1: low barrier; Session 2: medium barrier, Session 3: high barrier). There was never a barrier on the low reward arm to provide a consistently low-effort, low-reward option. Contrary to training, after choosing an arm, the rat was not blocked from traversing in the opposite direction to engage with the other arm containing the opposite 
barrier condition and opposite reward. However, after beginning to eat the chosen food reward, if the rat attempted to traverse the opposite direction it was blocked by the plastic partition, thereby requiring the rat to enter the center arm of the T-maze to begin another trial. Testing order of the rats was randomized within sets of approximately five animals per housing condition (i.e., $5 \mathrm{SH}$ tested, $5 \mathrm{NSH}$ tested, etc.) using an online random list generator (random.org).

Food obtainment (high reward vs. low reward) and perseverative behavior (defined as the time spent exploring the barrier) was recorded for each trial. Recording of perseverative behavior was restricted to the exact dimensions of the barrier; meaning perseverative behavior began when the rat came into contact with the barrier, including interacting with whiskers by sniffing. Perseverative behavior ceased when the rat cut contact, stopped sniffing, and began to move away from the barrier.

Analysis. Ten trials per condition were tested in order to minimize the possibility that behavioral results represented reinforcement-based learning and to maximize the possibility that they instead represented instinctive perseverance. For this reason, averages across ten trials per condition (i.e., barrier height) were analyzed; we were unable to evaluate trial-by-trial data due to inherent variability in such few trials. Proportions were arcsine transformed before statistical analysis to better approximate the normality assumption underlying parametric statistics (Keppel \& Wickens, 2004, p. 155). All t-tests were two-tailed. Latencies reported were medians for correct responses only. An alpha level of $p<.05$ was applied in all analyses.

\section{Results}

In training, on average, NSH rats obtained the high reward in $75.5 \%(S D=6.85 \%)$ of trials and SH rats obtained the high reward in $79.4 \%(S D=10.44 \%)$ of trials. There was no significant difference between the average amounts of high reward obtained between NSH and SH rats during training $(t(8)=$ $0.938, p=.376, d=0.313$ ). To assess task performance during testing, we used a $3 \times 2$ mixed-design analysis of variance (ANOVA) to examine the effect of barrier height and housing condition on the proportion of high reward obtained. There was a significant main effect of barrier height $(F(2,34)=$ $\left.40.12, p<.001, \eta_{\mathrm{p}}^{2}=0.70\right)$, no effect of housing condition, $\left(F(1,17)=0.493, p=.492, \eta_{\mathrm{p}}^{2}=0.03\right)$, and no significant interaction between barrier height and housing condition $\left(F(2,34)=1.09, p=.346, \eta_{\mathrm{p}}^{2}=\right.$ 0.06 , Figure 3A).

To explore the main effect of barrier height, post hoc analyses (paired samples $t$-test) revealed that all rats (collapsed across housing) were more successful at obtaining the high reward in the low barrier condition $(M=0.65, S D=0.14)$ relative to the medium barrier condition $(M=0.41, S D=0.22)$, $t(18)=5.73, p<.001$, as well as the high barrier condition $(M=0.31, S D=0.17), t(18)=8.60, p<.001$. There also was a significant difference between the high and medium barriers in terms of proportion high reward obtained, $t(18)=2.92, p=.009$. Thus, rats were most successful in obtaining the high reward when the barrier was lowest and success decreased as the barrier height increased.

We assessed perseverative behavior by analyzing the time spent interacting with each barrier (low, medium, high) as a function of housing condition (NSH; $\mathrm{SH}$ ) using a $3 \times 2$ mixed ANOVA for adaptive and maladaptive perseverative trials separately. Adaptive perseverative trials were considered those in which rats crossed the barrier and obtained its respective high reward, whereas maladaptive perseverative trials were considered those in which rats interacted with the high-reward barrier but eventually reverted to the low-effort, low-reward option. For adaptive perseverative trials (time spent interacting with barrier; high reward obtained), there was a significant main effect of barrier height, $F_{2,3}$ $=7.40, p=.002, \eta_{\mathrm{p}}^{2}=0.30$, Figure 3B. There was no effect of housing, $F(1,17)=0.07, p=.79, \eta_{\mathrm{p}}^{2}=$ 0.004 , and no interaction between barrier height and housing, $F(2,34)=0.24, p=.788, \eta_{\mathrm{p}}^{2}=0.01$. Collapsing across housing condition, post hoc analyses (paired samples $t$-tests) revealed that rats spent significantly more time interacting with the medium barrier $(M=10.79, S D=6.17)$ than either the low barrier $(M=5.58, S D=3.42), t_{18}=3.39, p=.003$, or the high barrier $(M=7.11, S D=4.74), t(18)=2.83$, $p=.011$. Time spent with the high and low barriers did not differ significantly for adaptive perseverative trials, $t(18)=1.21, p=.243$. 
Hemmer et al. 174
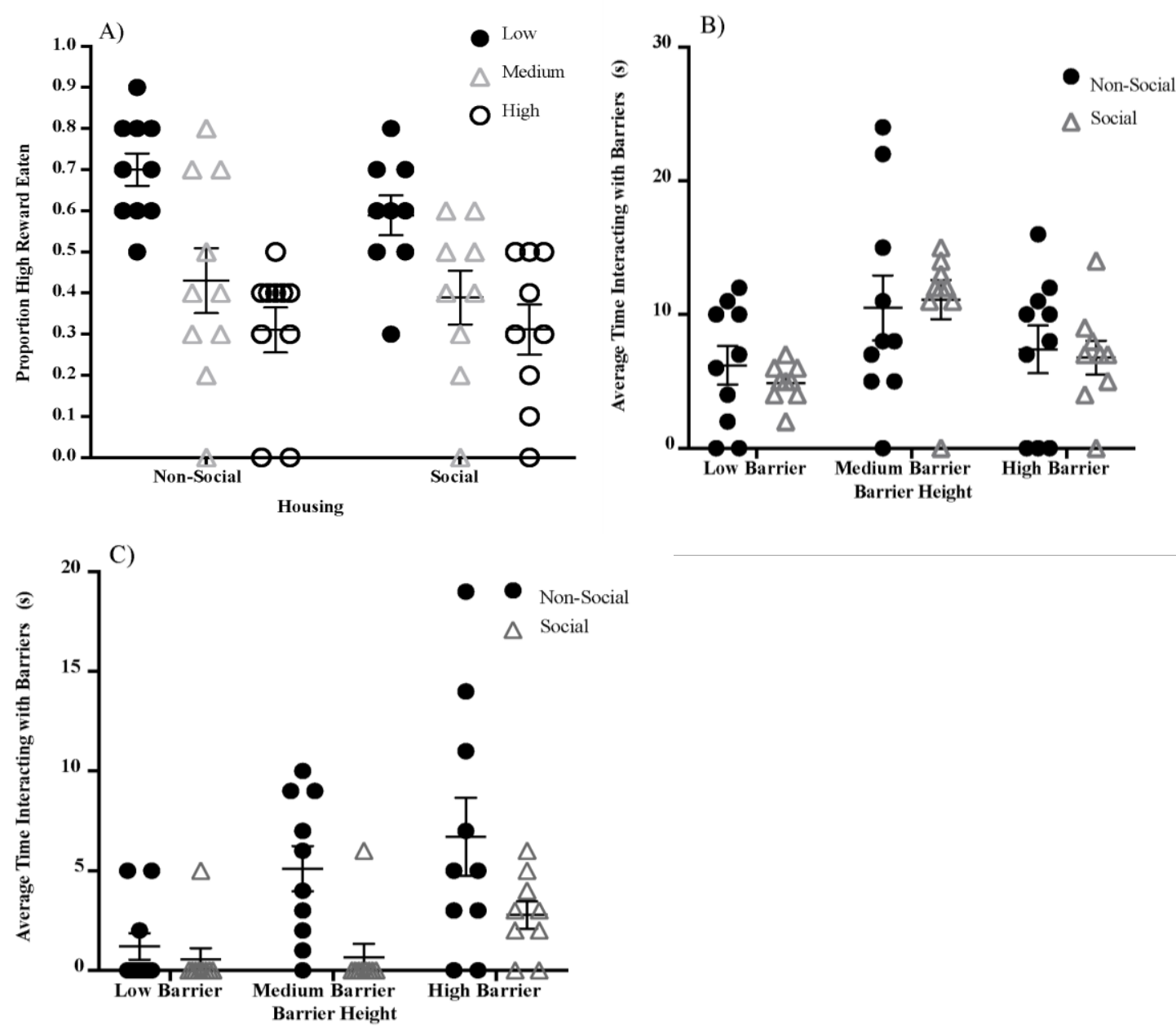

Figure 3. (A) Test performance (proportion high reward obtained) as a function of barrier height and social housing condition. Average time spent interacting with the barriers (in seconds) for (B) adaptive perseverative trials and (C) maladaptive perseverative trials in obtaining the high reward for nonsocially-housed rats and socially-housed rats. Each data point represents an individual rat; horizontal lines represent group averages; error bars represent standard errors of the mean. Asterisk denotes a statistically significant group difference $(p<.05)$.

For maladaptive perseverative trials (time spent interacting with barrier; low reward obtained after time spent at the high reward barrier), there was a significant main effect of barrier height, $F(2,34)$ $=6.70, p=.004, \eta_{\mathrm{p}}^{2}=0.28$, and of housing, $F(1,17)=9.37, p=.007, \eta^{2} \mathrm{p}=0.36$, (Figure $3 \mathrm{C}$ ). There was no interaction between barrier height and housing condition, $F(2,34)=1.89, p=.165, \eta_{\mathrm{p}}^{2}=0.10$. Collapsing across housing condition, post hoc analyses (paired samples $t$-tests) revealed that rats spent significantly more time interacting with the high barrier $(M=4.84, S D=5.01)$ than the low barrier $(M=$ $\left..895, S D=1.88 ; t_{18}=-3.45, p=.003\right)$ and the medium barrier $(M=3.00, S D=3.67)$ than the low barrier $\left(t_{18}=-2.46, p=.024\right.$. Time spent with the high and medium barriers did not differ significantly for maladaptive perseverative trials, $t(18)=-1.53, p=.144$.

Although we saw a significant difference in time spent interacting with the barriers during maladaptive perseverative trials, an independent samples $t$-test revealed no significant differences in the average number of adaptive perseverative trials and maladaptive perseverative trials between $\mathrm{SH}$ and $\mathrm{NSH}$ rats during any barrier height condition (adaptive: *medium: $t(8)=-1.512, p=.169, d=0.712$; high: $t(8)=0.555, p=.594, d=0.264$; maladaptive: low: $t(8)=1.00, p=.347, d=0.164$; medium: $t(8)=0.839$, 
$p=0.426, d=0.200$; high: $\mathrm{t}_{8}=0.610, p=.559, d=0.264 ;{ }^{*}$ note low adaptive could not be calculated because standard error of the difference is zero).

We also wanted to see if SH and NSH rats differed in the number of trials they chose the low reward option initially. A repeated measures ANOVA revealed that there was a significant main effect of barrier height $(F(2,34)=25.05, p=.000)$, no effect of housing condition, $(F(1,17)=0.003, p=.956)$, and no significant interaction between barrier height and housing condition $(F(2,34)=.254, p=.778)$. It is also important to note that rats infrequently chose the low reward arm initially and then switched to the high-effort, high-reward arm. Across barrier heights, NSH rats diverted from the low reward option to the high reward option only 6 times during the 300 total trials. Similarly, the SH rats switched from the low reward option to high reward option only 4 times out of 270 total trials.

Overall, there were no group differences in adaptive perseverative trials, but the NSH rats spent significantly more time interacting with the barriers in maladaptive perseverative trials compared to the SH rats.

\section{Discussion}

SH and NSH rats were equally proficient in overcoming a physical barrier to successfully obtain a higher-valued reward. Success varied systematically as a function of barrier height with the highest barrier leading to the lowest success rate and the lowest barrier leading to the highest success rate for all rats. Interestingly, perseverative behavior varied across NSH and SH rats. Although rats did not differ in time spent with the barriers on adaptive perseverative trials, NSH rats spent more time manipulating the barriers in maladaptive perseverative trials compared with $\mathrm{SH}$ rats.

Duration data for time spent interacting with the barrier suggest higher perseverative behavior in NSH rats compared with SH rats, but only when unsuccessful in climbing the barrier. NSH rats did not show increased perseverative behavior when successful in overcoming the barrier, indicating that they did not adaptively regulate their perseverative behavior to task demands. Interestingly, NSH rats expended more effort on a difficult task before reverting to the low reward option, considered maladaptive. Presumably, SH rats regarded this low-reward, effortless choice, as optimal earlier than NSH rats. SH rats therefore developed a more adaptable strategy, leading to less "wasted" time spent perseverating at the barrier before switching to the easier, more obtainable low food reward. In contrast, NSH rats reached this optimal decision of selecting the low-reward option during difficult trials only after maladaptively engaging with the barrier. We saw no significant difference in the number of times the rats opted to choose the low reward barrier at the beginning of the trial. This behavior could be considered advantageous because it did not result in "wasted" time at the barrier, but it is not indicative of maladaptive or adaptive perseverative behavior because these trials did not involve any perseveration.

The NSH rats' inability to adapt their perseverative behavior in this task and obtain rewards efficiently is consistent with other findings from our laboratory. In a separate experiment, SH rats refrain from maladaptive perseverative behaviors and acquire a task-set faster than NSH rats (Heimer-McGinn et al., submitted). In the Barnes maze, a goal box (GB) was hidden randomly under one of 18 possible holes that surrounded the periphery of a flat platform. Rats are uncomfortable in this open maze environment, making the escape to the GB a naturally rewarding behavior. SH rats demonstrated lower response latencies than NSH rats when traveling to a consistent GB location across trials. When, in a different iteration of the Barnes maze, the GB position changed randomly each trial, acquisition of task-set required cognitive flexibility in response to the changing task demands or rules. SH rats exhibited decreased perseverative errors compared with NSH rats. This could have partially contributed to a lower mean latency to reach the GB compared with NSH rats. Similar results were found with the same SH and $\mathrm{NSH}$ animals on a reversal task. In the current study, the perseverative behavior of the NSH rats was not considered a perseverative error because the high reward was always present if the rats overcame the barrier. However, the perseverative behavior of the NSH rats is considered maladaptive because it hindered their ability to abandon unsuccessful attempts to overcome the barrier or simply chose the low reward from the outset. Similar to the current study, perseverative behavior of NSH rats in our previous 
study resulted in erroneous re-visitations to un-baited locations, which may highlight a common inability of the NSH rats to adaptively regulate perseverative behavior.

Perseverance can positively or negatively affect an outcome depending on its probability of success. For example, a meta-analysis of perseverance revealed that high levels of perseverance are most useful when the task is difficult but well defined, indicating greater ability to succeed through sustained effort; however, perseverance can become counterproductive and maladaptive in tasks, especially in those that are ill-defined and present low potential to succeed (Credé et al., 2017). When human participants were asked to solve a set of anagrams, participants who scored higher on a measure of grit exhibited decreased performance due to their inability to give up on the unsolvable anagrams and instead complete problems that were solvable (Lucus et al., 2015). In this study, we show that nonsocial housing, or lack of social enrichment, can lead to maladaptive perseverative behavior. Maladaptive perseverative behavior of NSH animals could be explained by an underlying deficiency in acquiring a new task-set compared with SH animals. This heightened acquisition in SH rats may explain why they spent less time interacting with the barrier during maladaptive perseverative trials in the current study. As the barrier height increased, enhanced adaptability, and acquisition of task set in $\mathrm{SH}$ rats enabled them to respond advantageously. Essentially, SH rats "wasted" less time than NSH rats, spending less time on failed attempts to retrieve the high reward.

Previous work in our lab indicated that social housing confers advantageous decision-making (Heimer-McGinn et al., submitted), which makes it is the most likely candidate for the mechanism guiding the group differences observed in this study. However, it is possible that another aspect of sociality is responsible. For example, conflicts between conspecifics in the social housing condition could have resulted in less dominant rats learning to concede from the barrier earlier and switch to the low reward option. As a result of the NSH rats lacking interaction with conspecifics, they engaged in prolonged interaction with the barrier before reverting to the low reward. Further research should be conducted to better elucidate which aspect of social housing results in adaptive perseverative behavior.

To further delineate adaptive and maladaptive perseverance by making adaptive trials more advantageous and maladaptive trials even more aversive, future studies could limit the amount of time a rat has to complete a trial. In the current experimental design, rats were not restricted by time; trials would end once rats ate either reward. Thus, rats received a reward every trial. By adding a time constraint, perseverance at the high barrier could result in the trial ending without a reward and be considered even more maladaptive. Additionally, researchers could limit the total time for a session, such that maladaptively perseverating on trials resulted in a diminished net number of rewards gained.

Although it was deliberate to test animals on such few trials, future studies may also benefit from more testing trials in order to examine perseverative behavior over time and determine to what extent rats may modify their behavior based on previous trial outcomes. Various factors, including reinforcement contingencies, have been shown to influence the balance by which rats pursue a win-stay strategy compared to a win-shift strategy (Haig, Rawlins, Olton, Mead, \& Taylor, 1983). In the current experiment, observing the factors that influence when rats pursued a win-stay strategy compared to a winshift strategy would help elucidate the mechanism guiding rats to increase and/or cease perseverative behavior in order to achieve a positive outcome.

The negative or maladaptive aspect of perseverance commonly fits under the umbrella term "perseverative behavior". It is considered different from perseverance in the literature, as perseverance is often considered positive and similar to grit and even resilience. Yet perseverance and perseverative behavior may actually describe two sides of one common construct. To ascertain that sociality has a positive effect on perseverance, the results would have had to have shown that $\mathrm{SH}$ rats differentially perseverated on adaptive perseverative trials compared with NSH rats. Instead, because there was no difference in perseveration during adaptive perseverative trials, our results do not provide evidence for sociality positively influencing perseverance. However, because SH rats exhibited less maladaptive perseverance, thus, positively influencing adaptive decision-making, the conversation is directed toward the conclusion that lack of sociality increased perseverative behavior. The question still remains if social housing affects the positive aspects of perseverance, but the results of the current study suggest that social 
housing mitigates the maladaptive aspects of perseverative behavior, resulting in advantageous behavioral choices.

The current finding that social housing mitigates the maladaptive aspects of perseverative behavior was obtained in aged rats (22 months). Perseverative errors have been shown to increase with age in human and nonhuman primates (Head, Kennedy, Rodrigue, \& Raz, 2009; Lacreuse, Parr, Chennnareddi, \& Herndon, 2018) and are associated with smaller prefrontal cortex (PFC) volume and white matter integrity (Gunning-Dixon \& Raz, 2003; see meta-analysis by Yuan \& Raz, 2014). Thus, it may be possible that differences in perseverance between SH and NSH animals may be age-dependent and that perseverative behavior is more pronounced in aged NSH rats. This would be consistent with our laboratory's recent finding that social housing protected against an age-related decline in working memory errors in the radial arm maze (Templer et al., 2019). Differences in working memory errors and perseverative behavior observed here are likely to have common neural underpinnings in the PFC, an area that is particularly vulnerable to deterioration in old age (e.g., Wang et al., 2011). At the same time, it also is possible that differences in perseveration by SH and NSH rats is not age-dependent and might actually occur earlier in the lifespan. Increased acquisition of task set by SH rats in our previous study was evident in late adulthood and middle age (Heimer-McGinn et al., submitted). Unfortunately, because we did not test animals at an earlier age, we are unable to determine if the observed group differences are agedependent and share an underlying mechanism with the working memory system, or if differences in perseveration represent non-age-related acquisition benefits. We look forward to future research that tests animals at earlier ages to better dissociate the mechanisms underlying the cognitive benefits of social housing.

The current study is an important first step in understanding the complex construct of perseverance and offers an ideal platform to do so using animal models. Our experimental design allows for the isolation of adaptive and maladaptive perseverance to study the different facets of perseverance. Future studies could use our model to expand upon our findings and further isolate individual factors that influence both positive and negative aspects of perseverative behavior. Better isolation of these adaptive and maladaptive decisions could critically inform how these constructs are considered and classified in the human literature.

\section{Acknowledgments}

This work was supported by the NIH RI-INBRE (grants 2P20GM203430 and 2P20GM03430) and the Rhode Island Foundation (grant 20144297).

\section{References}

Charney, D. S. (2004). Psychobiological mechanisms of resilience and vulnerability: Implications for successful adaptation to extreme stress. American Journal of Psychiatry, 161, 195-216.

Cloninger, C. R., Zohar, A. H., Hirschmann, S., \& Dahan, D. (2012). The psychological costs and benefits of being highly persistent: Personality profiles distinguish mood disorders from anxiety disorders. Journal of Affective Disorders, 136, 758-766.

Credé, M., Tynan, M. C., \& Harms, P. D. (2017). Much ado about grit: A meta-analytic synthesis of the grit literature. Journal of Personality and social Psychology, 113, 492-511.

Domènech-Abella, J., Lara, E., Rubio-Valera, M., Olaya, B., Moneta, M. V., ...Haro, J. M. (2017). Loneliness and depression in the elderly: The role of social network. Social Psychiatry and Psychiatric Epidemiology, 52, 381-390.

Duckworth, A. L., Peterson, C., Matthews, M. D., \& Kelly, D. R. (2007). Grit: Perseverance and passion for longterm goals. Journal of Personality and Social Psychology, 92, 1087-1101.

Eisenberger, R. (1992). Learned industriousness. Psychological Review, 99, 248-267.

Feather, N. T. (1962). The study of persistence. Psychological Bulletin, 59, 94-115.

Feder, A., Nestler, E. J., \& Charney, D. S. (2009). Psychobiology and molecular genetics of resilience. Nature Reviews Neuroscience, 10, 446-457. 
Fone, K. C. F., \& Porkess, M. V. (2008). Behavioural and neurochemical effects of post-weaning social isolation in rodents - Relevance to developmental neuropsychiatric disorders. Neuroscience \& Biobehavioral Reviews, 32, 1087-1102.

Garcia, D. (2011). Two models of personality and well-being among adolescents. Personality and Individual Differences, 50, 1208-1212.

Gunning-Dixon, F. M., \& Raz, N. (2003). Neuroanatomical correlates of selected executive functions in middleaged and older adults: a prospective MRI study. Neuropsychologia, 41, 1929-1941.

Haig, K. A., Rawlins, J. N. P., Olton, D. S., Mead, A., \& Taylor, B. (1983). Food searching strategies of rats: Variables affecting the relative strength of stay and shift strategies. Journal of Experimental Psychology: Animal Behavior Processes, 9, 337-348.

Head, D., Kennedy, K. M., Rodrigue, K. M., \& Raz, N. (2009). Age differences in perseveration: Cognitive and neuroanatomical mediators of performance on the Wisconsin Card Sorting Test. Neuropsychologia, 47, 1200-1203.

Heim, C., Newport, D. J., Mletzko, T., Miller, A. H., \& Nemeroff, C. B. (2008). The link between childhood trauma and depression: Insights from HPA axis studies in humans. Psychoneuroendocrinology, 33, 693-710.

Heimer-McGinn, V. R., Wise, T. B., Hemmer, B. M., Dayaw, J. N. T., Templer, V. L. (2019). Social housing enhances acquisition of task set independently of environmental enrichment: A longitudinal study in the Barnes maze. Manuscript submitted for publication.

Keppel, G., \& Wickens, T. D. (2004). Design and analysis, a researcher's handbook (4th ed.). Upper Saddle Rober, NJ: Pearson.

Lacreuse, A., Parr, L., Chennareddi, L., \& Herndon, J. G. (2018). Age-related decline in cognitive flexibility in female chimpanzees. Neurobiology of Aging, 72, 83-88.

Lambert, K. G., Tu, K., Everette, A., Love, G., McNamara, I., ... Kinsley, C. H. (2006). Explorations of coping strategies, learned persistence, and resilience in Long-Evans rats: Innate versus acquired characteristics. Annals of the New York Academy of Sciences, 1094, 319-324.

Laurence, N. C., Labuschagne, L. G., Lura, B. G., \& Hillman, K. L. (2015). Regular exercise enhances task-based industriousness in laboratory rats. PLOS ONE, 10, e0129831.

Lucas, G. M., Gratch, J., Cheng, L., \& Marsella, S. (2015). When the going gets tough: Grit predicts costly perseverance. Journal of Research in Personality, 59, 15-22.

Mukerjee, S. (2013). An empirical analysis of the association between social interaction and self-rated health. American Journal of Health Promotion, 27, 231-239.

Rasmusson, A. M., Vythilingam, M., \& Morgan, C. A. (2003). The neuroendocrinology of posttraumatic stress disorder: New directions. CNS Spectrums, 8, 651-667.

Rayburn-Reeves, R. M., \& Cook, R. G. (2016). The organization of behavior over time: Insights from mid-session reversal. Comparative Cognition \& Behavior Reviews, 11, 103-125.

Russo, S. J., Murrough, J. W., Han, M.-H., Charney, D. S., \& Nestler, E. J. (2012). Neurobiology of resilience. Nature Neuroscience, 15, 1475-1484.

Simpson, J., \& Kelly, J. P. (2011). The impact of environmental enrichment in laboratory rats-Behavioural and neurochemical aspects. Behavioural Brain Research, 222, 246-264.

Templer, V. L., Wise, T. B., Dayaw, K. I. T., \& Dayaw, J. N. T. (2018). Nonsocially housed rats (Ratus norvegicus) seek social interactions and social novelty more than socially housed counterparts. Journal of Comparative Psychology, 132, 240.

Templer, V. L., Wise, T. B., \& Heimer-McGinn, V., (2019) Social housing decreases working memory errors but not reference memory errors in aged rats. Neurobiology of Aging, 75, 117-125.

Wang, M., Gamo, N. J., Yang, Y., Jin, L. E., Wang, X. J., ...Arnsten, A. F. (2011). Neuronal basis of age-related working memory decline. Nature, 476, 210.

Williams, L. A., \& DeSteno, D. (2008). Pride and perseverance: The motivational role of pride. Journal of Personality and Social Psychology, 94, 1007-1017.

Yuan, P., \& Raz, N. (2014). Prefrontal cortex and executive functions in healthy adults: A meta-analysis of structural neuroimaging studies. Neuroscience \& Biobehavioral Reviews, 42, 180-192. 